This article was downloaded by: [François Bonnetblanc]

On: 13 July 2011, At: 05:09

Publisher: Psychology Press

Informa Ltd Registered in England and Wales Registered Number: 1072954 Registered office: Mortimer House, 37-41 Mortimer Street, London W1T 3J H, UK

\title{
Neurocase
}

Publication details, including instructions for authors and subscription information: http:// www. tandfonline.com/loi/ nncs20

\section{Ultra-fast recovery from right neglect after 'awake surgery' for slow-growing tumor invading the left parietal area}

\author{
Etienne Sallard $^{a b}$, Hugues Duffau ${ }^{c d} \&$ François Bonnetblanc ${ }^{a b}$ \\ a Université de Bourgogne, Dijon, Campus Universitaire, Unité de Formation et de \\ Recherche en Sciences et Techniques des Activités Physiques et Sportives, Dijon, \\ France \\ ${ }^{b}$ Institut National de la Santé et de la Recherche Médicale, Unité 887, Motricité- \\ Plasticité, Dijon, France \\ ' Département de Neurochirurgie, Hôpital Gui de Chauliac, Centre Hospitalier \\ Universitaire Montpellier, France \\ d Institut National de la Santé et de la Recherche Médicale, Unité 1051, Institut des \\ Neurosciences de Montpellier, Montpellier, France
}

Available online: 13 J uly 2011

To cite this article: Etienne Sallard, Hugues Duffau \& François Bonnetblanc (2011): Ultra-fast recovery from right neglect after 'awake surgery' for slow-growing tumor invading the left parietal area, Neurocase, DOI: $10.1080 / 13554794.2011 .556127$

To link to this article: http:// dx. doi.org/ 10.1080/ 13554794.2011.556127

\section{iFirst}

\section{PLEASE SCROLL DOWN FOR ARTICLE}

Full terms and conditions of use: http://www.tandfonline.com/page/terms-and-conditions

This article may be used for research, teaching and private study purposes. Any substantial or systematic reproduction, re-distribution, re-selling, loan, sub-licensing, systematic supply or distribution in any form to anyone is expressly forbidden.

The publisher does not give any warranty express or implied or make any representation that the contents will be complete or accurate or up to date. The accuracy of any instructions, formulae and drug doses should be independently verified with primary sources. The publisher shall not be liable for any loss, actions, claims, proceedings, demand or costs or damages whatsoever or howsoever caused arising directly or indirectly in connection with or arising out of the use of this material. 


\title{
Ultra-fast recovery from right neglect after 'awake surgery' for slow-growing tumor invading the left parietal area
}

\author{
Etienne Sallard $^{1,2}$, Hugues Duffau ${ }^{3,4}$, and François Bonnetblanc ${ }^{1,2}$ \\ ${ }^{1}$ Université de Bourgogne, Dijon, Campus Universitaire, Unité de Formation et de Recherche en Sciences \\ et Techniques des Activités Physiques et Sportives, Dijon, France \\ ${ }^{2}$ Institut National de la Santé et de la Recherche Médicale, Unité 887, Motricité-Plasticité, Dijon, \\ France \\ ${ }^{3}$ Département de Neurochirurgie, Hôpital Gui de Chauliac, Centre Hospitalier Universitaire \\ Montpellier, France \\ ${ }^{4}$ Institut National de la Santé et de la Recherche Médicale, Unité 1051, Institut des Neurosciences de \\ Montpellier, Montpellier, France
}

\begin{abstract}
It is now possible to perform resections of slow-growing tumors in awake patients. Using direct electrical stimulation, real-time functional mapping of the brain can be used to prevent the resection of essential areas near the tumor. Simple clinical observations of patients with a resection of slow-growing tumors have demonstrated substantial recovery within a few days of such 'awake surgery'. The aim of this study was to investigate the kinetics of recovery following the resection of slow-growing tumors invading the left parietal area and to focus mainly on its rapidity. Two patients were assessed by standard line bisection tests and compared with eight healthy individuals. Independently of the pure nature of the symptoms, we report that the patients rapidly and substantially recovered from pronounced right neglect. They were tested 48 hours after the surgery and the recovery was significant for both patients after less than 4 hours. Strikingly, for one patient, recovery was ultra fast and substantial in the first practice session within less than 7 minutes: it occurred without verbal feedback and was substantially retained during the following testing session. Its rapidity suggests a process of unmasking redundant networks. With the slow growth of the lesion, the contralesional hemisphere is probably progressively prepared for rapid unmasking of homologue networks. These results have major clinical implications. For patients with an invading left-side tumor, it is now clear that line bisections are required before, during, and after awake surgery to: plan the surgery, control the quality of the resection, and also optimize the rehabilitation of the patient.
\end{abstract}

Keywords: Plasticity; Slow-growing tumor; Right hemineglect; Awake surgery.

Functional recovery is considerably better in the context of slow-growing injuries than after acute lesions. The recruitment of remote brain areas in the ipsi- and contralesional hemispheres is much more efficient in slow growing than acute lesions.
Thus, the contrasting recoveries and plastic processes associated with the two types of lesion offer interesting insights that may help understand brain plasticity (Duffau, 2005; Bonnetblanc, Desmurget, \& Duffau, 2006; Desmurget, Bonnetblanc, \&

This study was supported by the 'Association pour le Recherche sur le Cancer' (ARC-France Subvention Libre no. 3184). We gratefully thank the patients for their participation to this study. We also thank the two anonymous reviewers for their stimulating comments about the present work.

Address correspondence to François Bonnetblanc, Institut National de la Santé et de la Recherche Médicale, Unité 887, MotricitéPlasticité, F-21078 Dijon, France. (E-mail: francois.bonnetblanc@u-bourgogne.fr).).

(C) 2011 Psychology Press, an imprint of the Taylor \& Francis Group, an Informa business 
Duffau, 2007; Finger, 1978; Finger \& Stein, 1982). For slow-growing tumors, the impressive reorganization capacity of the brain allows the subjects to recover rapidly, within a few days after surgery (Bonnetblanc et al., 2006; Desmurget et al., 2007; Duffau, 2005). However, although there is clinical evidence, there has been no precise quantification of these phenomena. We report an analysis of the kinetics of recovery following surgery for low-grade gliomas (LGG WHO Grade II gliomas).

LGG are slow-growing tumors of the CNS. They progress slowly $(4 \mathrm{~mm}$ per year on average Mandonnet et al., 2003) for 7-8 years, before undergoing anaplastic transformation. During this slow progression, most LGG patients exhibit normal clinical exam results (Walker \& Kaye, 2003), although mild cognitive disorders can be detected by extensive assessment (Taphoorn \& Klein, 2004). LGG resection is subject to two constraints: (i) removing as much of the invaded tissue as possible and (ii) minimizing functional sequelae. The use of direct electrical stimulation (DES) is widely advocated during surgical resections (Berger \& Rostomily, 1997; Duffau, Bauchet, Lehericy, \& Capelle, 2001; Duffau, 2005; Duffau et al., 2003a; Duffau, Gatignol, Mandonnet, Capelle, \& Taillandier, 2008; Keles \& Berger, 2004; Keles et al., 2004; Sanai, Mirzadeh, \& Berger, 2008). This technique causes a transient virtual lesion, thus allowing the structures that are essential for function to be identified at each stage of the resection (Duffau, 2004). The rules guiding this approach are simple: any reproducible functional disturbance induced by DES indicates interruption of the resection (Duffau, 2005; Duffau et al., 2008). This technique has proved to be effective: more than $90 \%$ of the patients return to normal socio-professional life, with no functional deficit, only 3 months after the surgery. Some very basic clinical observations indicate that in some cases patients could recover substantially within a single day (Duffau, 2001, 2008; Duffau \& Capelle 2001a, 2001b; Duffau, Sichez, \& Lehericy, 2000; Krainik et al., 2003). However, the kinetics of this recovery have not been sufficiently described. Clinical observations reported are at single time points and most are based on global neurological and rating scales.

We report two patients tested after resection of their left parietal area invaded by a LGG, to measure their visuo-attentional deficits. Neglect is most often associated with right-side brain damage (Kandel, Schwartz \& Jessell, 2000; Purves et al., 2008; Robertson \& Marshall, 1993). By contrast, right neglect (left-side lesions) has been observed in stroke patients with various frequencies and severity. They are less often observed (0-25\% for left vs. $33-85 \%$ for right hemisphere strokes), less severe and recover more frequently than right lesions ( $60 \%$ for left vs. $0-50 \%$ for right strokes) (Bowen, McKenna, \& Tallis, 1999; Chain, Leblanc, Chedru, \& L'Hermitte, 1979; Chedru, Leblanc M, L'Hermitte, 1973; Ogden, 1985; Stone et al., 1991). More specifically, for stroke patients with left brain damages, Denes, Semenza, Stoppa, and Lis (1982), Sunderland, Wade, and Langton-Hewer (1987), and Stone et al. (1991) observed a substantial recovery of right neglects after at least 3 months. These data suggest that even if right neglects usually occur less frequently and the severity level is also lower than left neglect, the recovery remains also quite long after acute lesions. In any case, recovery for right neglect was never observed within a single day, before the present work.

We used a classical line bisection task to quantify the visuo-attentional deficit for both patients and to determine how rapidly they recovered after the surgery. Line bisection is a common procedure used in LGG surgery. Intra-operative line bisection tasks combined with DES have been successfully used to track the visuo-attentional network involved in the symmetrical processing of visual scenes for patients with a right lesion (Thiebaut de Schotten et al., 2005). However, visuo-attentional deficits affecting patients with left lesions have been less extensively explored. We found out that both patients displayed severe right neglect. However, 48 hours after the surgery both patients were tested and this deficit improved within a few minutes only as assessed by a simple line bisection task without verbal feedback.

\section{METHODS}

\section{Subjects}

Two right-handed patients were compared with eight right-handed healthy subjects (Controls). The control subjects (five men and three women) were aged from 37 to 56 years and had no history of neurological disease; they all participated in the study on a voluntary basis according to university protocols.

The two patients: P1 was a 39-year-old woman and $\mathrm{P} 2$ was a 41-year-old man. Both underwent surgical resection of a low-grade glioma (WHO II) using intra-operative DES (Duffau et al., 2005). 
(a)

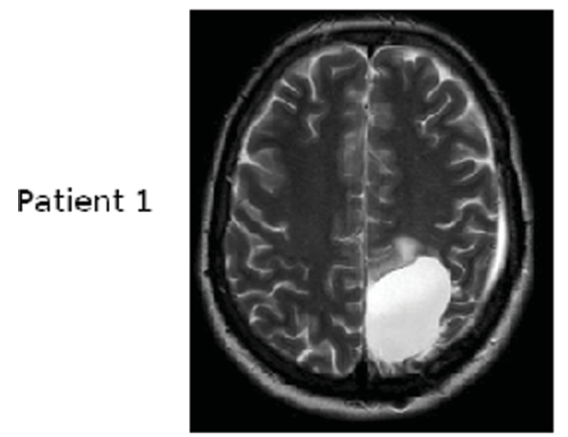

MRI post-resection
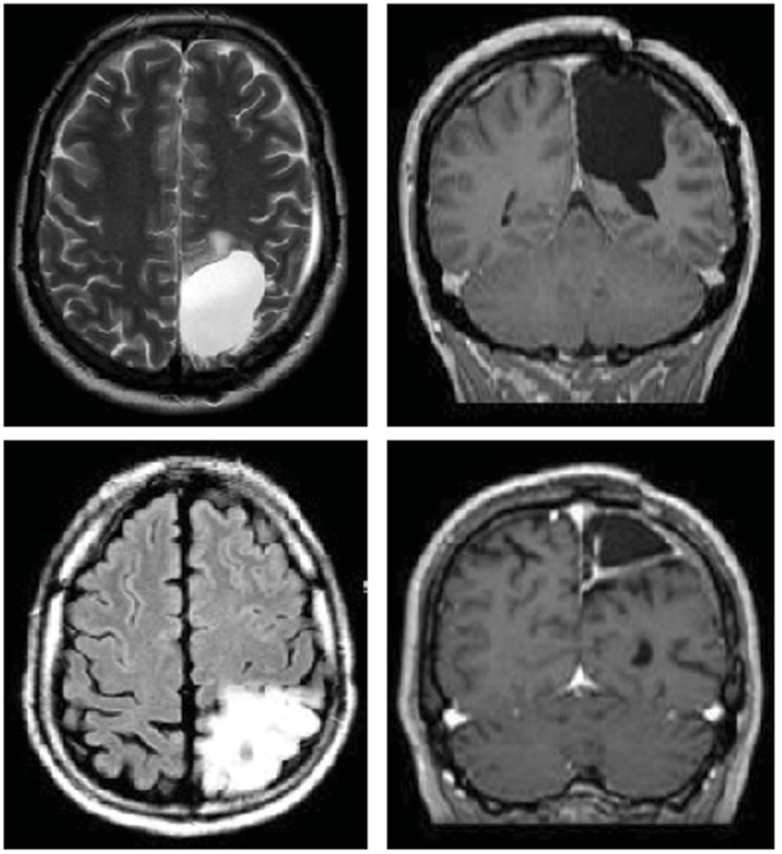

MRI post-resection
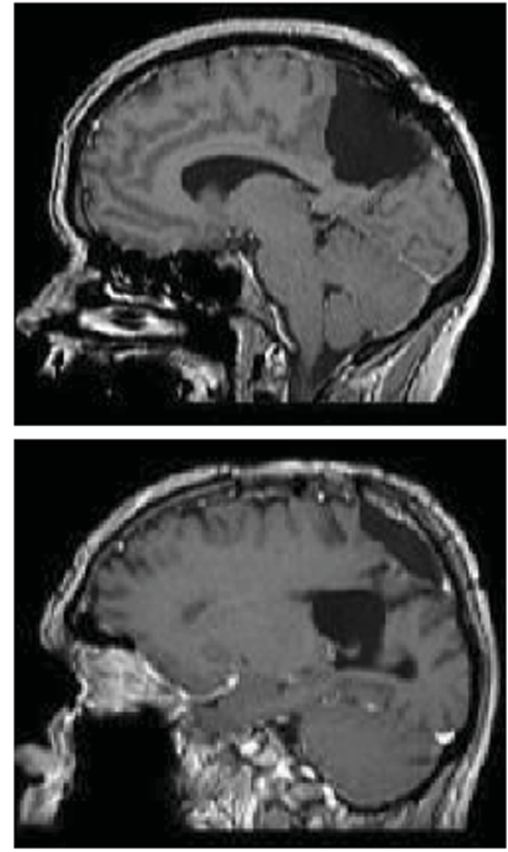

(b)

Intra-operative pre-resection

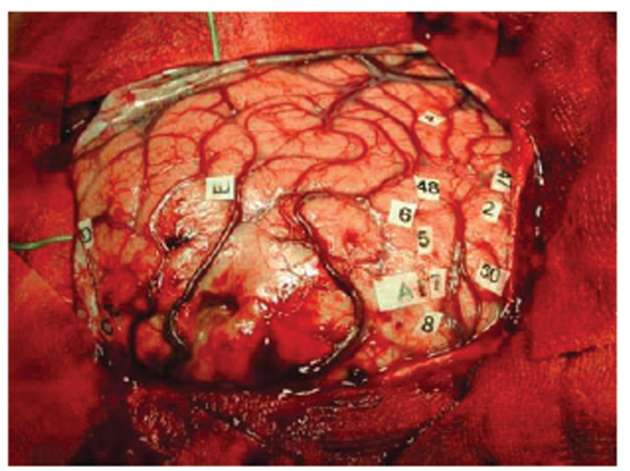

Patient 1

Patient 2

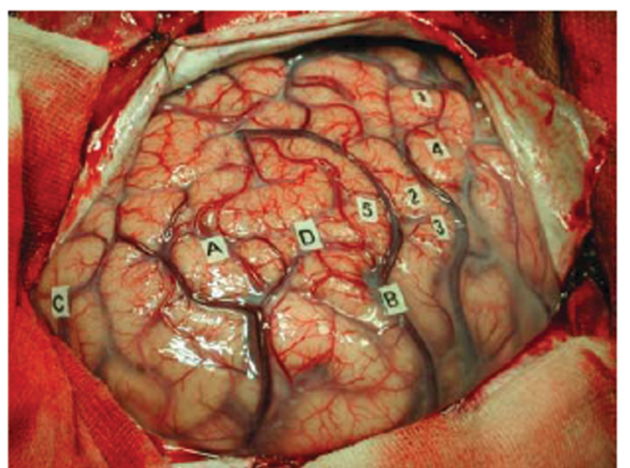

Intra-operative post-resection
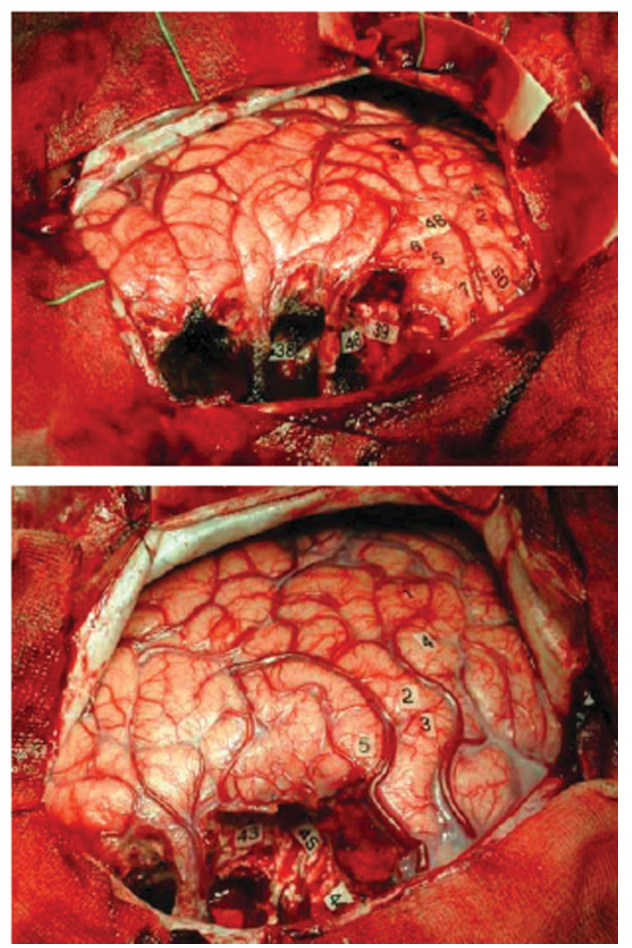

Figure 1. (a) Pre-operative axial T2/FLAIR-weighted MRI views and post-operative coronal and saggital views of patients P1 (upper panel) and P2 (lower panel) obtained by T1-weighted MRI (neurological convention), showing tumor invasion and resection of the left parietal area. (b) Pictures of the functional mapping of the area and subcortical pathways before and after resection in patients P1 (upper panel) and P2 (lower panel). 
P1 and P2 were put under clinical observation because of epileptic seizures. Interestingly, during the medical consultation, both reported transient visuo-motor impairments preceding the seizures, and dysesthesia. However, consistent with the slowly infiltrative character of the lesion, preoperative neurological examination revealed no deficit, or any visuo-motor abnormality, in either patient. In both $\mathrm{P} 1$ and $\mathrm{P} 2$, pre-operative MRI confirmed that the glioma was centered on the left parietal area (Figure 1). The initial volumes of the tumors were estimated to be approximately $90 \mathrm{~cm}^{3}$ in $\mathrm{P} 1$ and $50 \mathrm{~cm}^{3}$ in $\mathrm{P} 2$.

\section{Surgical procedure}

Patients were placed in a lateral position on their right side. Intra-operative mapping was performed under local anesthesia using the direct electrical stimulation technique as previously described (Duffau et al., 2005, 2008). Briefly, a bipolar electrode with $5-\mathrm{mm}$ spaced tips delivering a biphasic current not deleterious for the nervous system (pulse frequency of $60 \mathrm{~Hz}$, single pulse phase duration of $1 \mathrm{~ms}$, amplitude from 2 to $6 \mathrm{~mA}$ ) was placed on the patient's brain while awake. The stimulation began 0.5 second before the presentation of the cross-modal stimuli and lasted 1 second for sensory-motor mapping and 4 seconds for language mapping.

First, motor and somatosensory functions were assessed. In resting patients, stimulation was administered to the central area, and this induced involuntary motor responses; stimulations were applied so as to detect the motor sites and somatosensory sites which elicited the dysesthesia described by the patients. Second, the patients were asked to count (repetitively from 1 to 10 ) to identify the essential areas for speech production, namely those resulting in complete anarthria when stimulated. Finally, a picture-naming test (DO 80) consisting of 80 black and white drawings of objects was used to detect anomia and naming impairment.

The patient, the speech therapist, and the experimenter were all blind as to when the brain was stimulated. Each site was tested at least three times, three trials being enough to establish whether or not a cortical site is essential for a particular function (Ojemann, Ojemann, Lettich, \& Berger, 1989). To avoid seizures, the same cortical site was never stimulated twice in a row. Moreover, to ensure successful tumor removal while sparing functional areas, the limits of the resection were progressively set using direct subcortical stimulation throughout the procedure so as to preserve functional white matter pathways in the immediate vicinity of the surgical cavity. This strategy minimizes residual morbidity while optimizing resection quality (Duffau, 2005).

\section{Post-operative behavioral task: line bisections}

The task consisted of bisecting lines of three different lengths in a random order (10 trials for each line length, i.e., 30 trials in all in each one session). P1 performed the task only with the right hand. P2 performed the task with the right hand and then with the left hand. The lines were presented at a reading distance on a landscape A4 sheet of white paper aligned with the sagittal midline of the subject. The lines were 50,150, and $250 \mathrm{~mm}$ long and $0.5 \mathrm{~mm}$ thick. Lines were totally randomized within each session and the presentation sequence was different for each participant. No verbal feedback about performance was given. Line bisection errors were measured with a precision of $0.5 \mathrm{~mm}$. Positive values indicate a rightward deviation and negative values a leftward deviation.

P1 was tested three times during the day starting 48 hours after the surgery and once 144 hours after surgery. For P1, 50, and $150 \mathrm{~mm}$ line lengths were not presented in every session. P2 was tested twice during the day starting 48 hours after the surgery and once 96 hours after surgery.

\section{RESULTS}

Importantly, the line bisection test we employed was not commonly used for patients with an invasion of the left parietal area before the present work. This test was mostly used to assess patients undergoing surgery for slow-growing tumors in the right parietal area and monitor visuo-attentional function during such surgery. This procedure clearly limited the functional deficits from right brain damage. Our two patients were the first such to experience this method. In consequence, these tests were only performed after the surgery for these two patients. For ethical reasons it seems now interesting to perform line bisection tests even for patients with an invasion of the left parietal cortex, in order to limit as much as possible the attentional deficit. However, it is difficult to say whether this new procedure will allow sparing new functional sites during the surgery as these patients are quite rare 
and because we have not yet collected data for line bisection tests performed during the surgery.

\section{Intra-operative results}

Patient 1: During the surgical session, the electrical stimulation in the primary motor area (precentral gyrus) produced hand (50) and face (2) movements; anarthria with stimulation in the rolandic operculum (47); dysesthesia of the face (4), fingers $(48,6,5,7)$, and of the forearm (8) with stimulation in the primary somatosensory area (retrocentral gyrus). No other response was induced by the stimulation of the other cortical sites (Figure 1b upper and left panel).

Resection in the subcortical area was also stopped according to functional boundaries, namely the thalamo-cortical somatosensory pathways of the upper limb in 39 (anterior and deep wall of the cavity) and of the lower limb in 46. The deep and lateral boundary of the resection was the superior longitudinal fasciculus, inducing phonemic paraphasia when stimulated (38) (Figure $1 \mathrm{~b}$ upper and right panel).

Patient 2: During surgery, electrical stimulation in the primary motor area (precentral gyrus) produced face movements (4); anarthria with stimulation in the rolandic operculum (precentral part in 1); dysesthesia of the fingers $(2,3)$ and of the hand (19) with stimulation in the primary somatosensory area (retrocentral gyrus). Stimulation of a discrete area of the superior and anterior part of the supramarginal gyrus elicited reproducible leftward deviation of the arm flexion (5) (Figure 1b lower and left panel).

At the subcortical level, the resection was again stopped according to functional boundaries, namely the thalamo-cortical somatosensory pathways of the lower limb in 44 (anterior and mesial the entire cavity) and upper limb in 45 (deep and lateral boundary of the resection). In addition, subcortical language pathways were identified more laterally and posteriorly, i.e., the postero-superior loop of the superior longitudinal fasciculus eliciting paraphasia when stimulated (in 43) (Figure 1b lower and right panel).

\section{Post-operative course}

Post-operative MRI showed a subtotal removal of the left superior parietal lobule in both cases, with a residual tumor estimated at $4 \mathrm{~cm}^{3}$ in both patients. The histological examination revealed a WHO grade II glioma in both cases.

\section{Post-operative behavioral and clinical assessments}

Despite a lesion in the left parietal area and according to Liepmann's model (Liepmann, 1920), no sign of apraxia was noticeable for either patient. They were not impaired when required to act out cutting a slice of bread, or opening a door with a key. In addition, there was no sign of hemianopsia, but there was evidence of optic ataxia: they were impaired when they had to grasp for an object presented from behind into their right peripheral visual field and without fovealization of this object.

However, just before performing the bisection task, during simple behavioral and clinical examinations, $\mathrm{P} 2$ presented some difficulties when spontaneously using his right hand especially for brushing his teeth or eating: he had a strong tendency to use his left hand rather than his right. Similarly, he was impaired when going through doorways and systematically bumped his right shoulder.

No such immediate behavioral evidence of right hemineglect was reported for P1 before performing the bisection task. However, during the second session of the bisection task, she suddenly reported: 'It's strange, but I didn't notice that my hand could mask the line' clearly suggesting right neglect during the practice session: she was not aware of her hand. Interestingly, despite spontaneously observing this problem, she still continued to make major errors (please see Figure 2).

\section{Line bisections}

Mean values for the patients were compared with the $95 \%$ confidence intervals (CI) determined for the control subjects (Table 1). During every testing session and for all line lengths the patients' score were outside these CIs. For medium and long lines, the patients systematically showed an abnormal mean leftward bias. By contrast, the patients systematically showed an abnormal mean rightward bias for small lines, revealing a significant crossover effect. The mean time taken to perform the line bisection task was 2 min $59 \mathrm{~s} \pm 56 \mathrm{~s}$ for the control subjects. For the patients this time was reported on Figures 2 and 3.

Normal ranges for line bisection were calculated on the overall performances across subjects. 


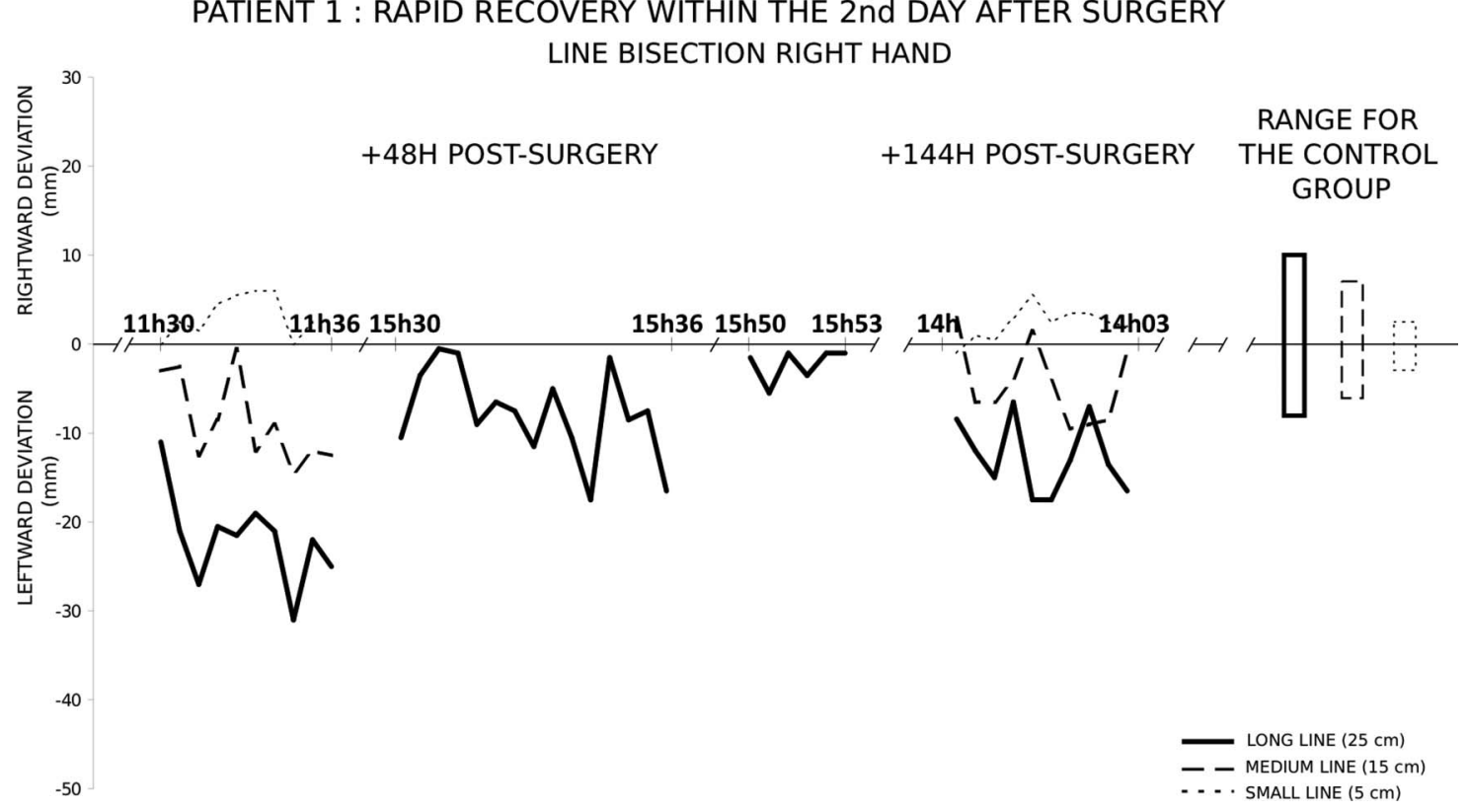

Figure 2. Deviations from the middle of the line at various times after surgery and for various line lengths for Patient 1 . Line bisection tests were performed with the right hand, 48 and 144 hours after awake surgery of the left parietal area. The rectangle on the right side of the figure represents the full range of deviations for control subjects, according to line lengths. Normal ranges for line bisection were calculated on the overall performances across subjects.

TABLE 1

Mean $\pm S D$ in the line bisection test performed with the right hand for Patient 1 and Patient 2 for various line length conditions and during different test sessions

\begin{tabular}{|c|c|c|c|c|c|c|c|c|}
\hline & \multicolumn{4}{|c|}{$\begin{array}{l}\text { Deviations for Patient } 1 \\
\text { Mean values in } m m \pm S D\end{array}$} & \multicolumn{3}{|c|}{$\begin{array}{l}\text { Deviations for Patient } 2 \\
\text { Mean values in } m m \pm S D\end{array}$} & \multirow{3}{*}{$\begin{array}{c}95 \% \\
\text { Confidence } \\
\text { interval for } \\
\text { control } \\
\text { subjects } \\
\text { (values are in } \\
\mathrm{mm} \text { ) }\end{array}$} \\
\hline & & $+48 h$ & & $+144 h$ & & $48 \mathrm{~h}$ & $+96 h$ & \\
\hline & $11: 30 \mathrm{~h}$ & $15: 30 \mathrm{~h}$ & $15: 50 \mathrm{~h}$ & $14 \mathrm{~h}$ & $12 \mathrm{~h}$ & $16 h$ & $14 \mathrm{~h}$ & \\
\hline Small (50 mm) & $2.95 \pm 2.26$ & & & $2.3 \pm 1.72$ & $1.85 \pm 7.67$ & $6.7 \pm 6$ & $3.7 \pm 1.96$ & {$[-0.6 ; 0.35]$} \\
\hline $\begin{array}{l}\text { Medium } \\
\quad(150 \mathrm{~mm})\end{array}$ & $-8.7 \pm 4.72$ & & & $-4.45 \pm 4.17$ & $-7.2 \pm 18.05$ & $-5.35 \pm 12.37$ & $-2.85 \pm 7.27$ & {$[-0.54 ; 2.06]$} \\
\hline $\begin{array}{l}\text { Long } \\
\qquad(250 \mathrm{~mm})\end{array}$ & $-21.9 \pm 4.99$ & $-7.8 \pm 4.95$ & $-2.25 \pm 1.7$ & $-12.65 \pm 4$ & $-19.65 \pm 17.32$ & $-1.35 \pm 13.38$ & $-7.35 \pm 7.7$ & {$[-0.45 ; 1.27]$} \\
\hline
\end{tabular}

These values are compared to the $95 \%$ CI obtained for the control subjects.

Both patients showed substantial recovery over time for the bisection of long lines: from 48 to 144 hours after the surgery $t(18)=-4.3368$, $p<.001$ for Patient 1 and from 48 to 96 hours $t(18)=1.95, p=.067$ for Patient 2 (single tail analysis). For Patient 1, the recovery was also a decrease of leftward deviations for the bisection of medium lines $(t(18)=2.024, p=.058$, for a single tail analysis). This was not observed for Patient $2(t(18)=0.67, p=.51)$, however, for this patient the variability during the first session was greater than that during the last session. For the bisection of small lines, mean performances were similar during the two different days of testing for each Patient $1(t(18)=0.6859$, $p>.05)$ and Patient $2(t(18)=-0.7005, p=.5)$. 
PATIENT 2 : RAPID RECOVERY WITHIN THE 2nd DAY AFTER SURGERY

LINE BISECTION RIGHT HAND

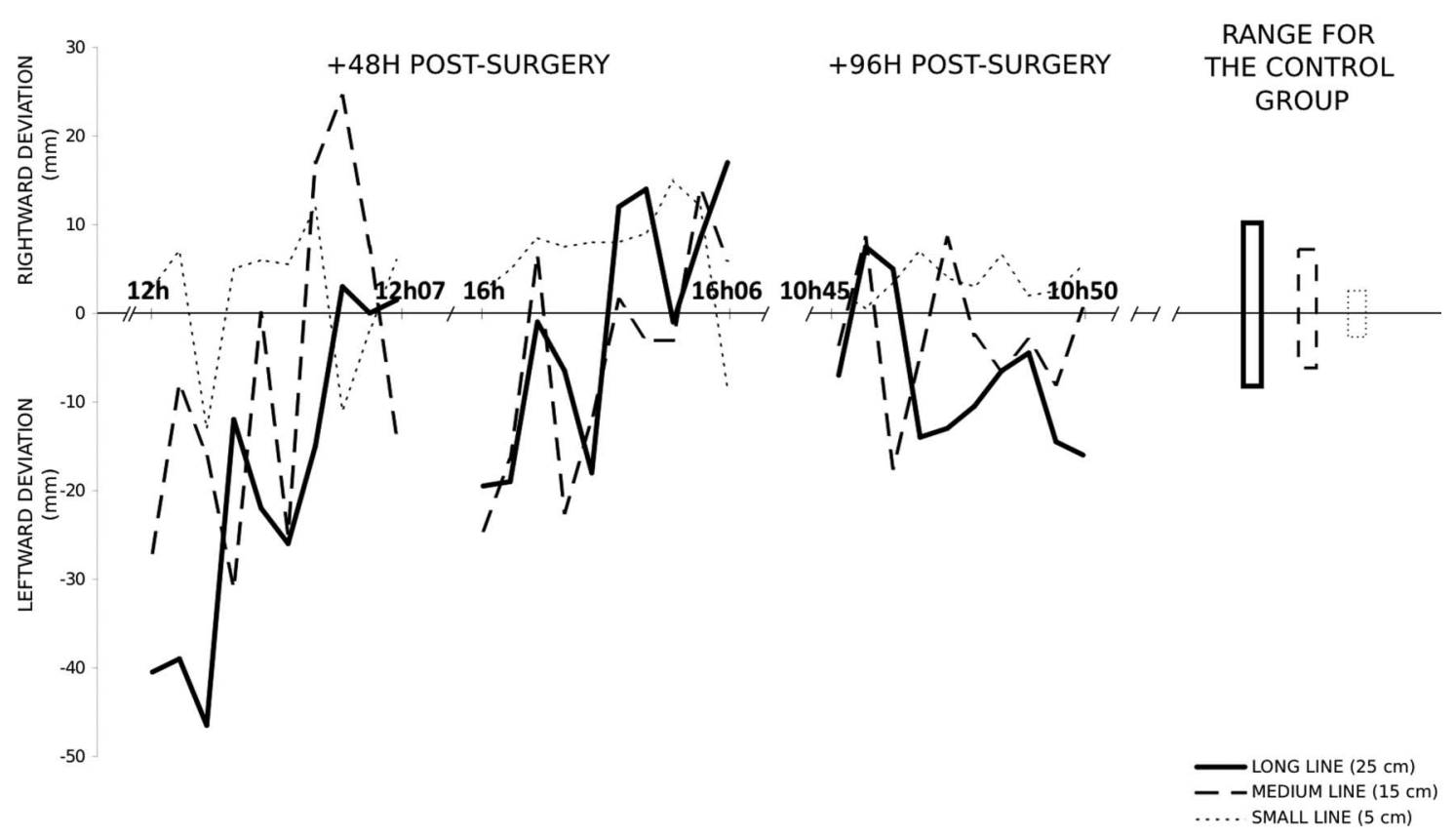

Figure 3. Deviations from the middle of the line at various times after surgery and for various line lengths for Patient 2. Line bisection tests were performed with the right hand, 48 and 96 hours after awake surgery of the left parietal area. The rectangle on the right side of the figure represents the full ranges of deviations for control subjects, according to line lengths. Normal ranges for line bisection were calculated on the overall performances across subjects.

Both patients underwent repeat testing, at 4-hour intervals, during the day 48 hours after surgery. They significantly improved their performances for the bisection of long lines between test sessions, $F(2,10)=24.5, p<.001$ for Patient 1 and $t(18)=-2.5071, p<.05$ for Patient 2. This was not observed for medium and small lines for Patient 2 ( $P$ values $>.1)$. However, for this patient the variability of deviations was greater during the first session than the second for the medium and small lines. Patient 1 explicitly reported substantial fatigue during the testing, especially the first and second sessions.

During the first session of testing, Patient 2 showed substantial recovery after only 7 minutes of practice: he started with a leftward deviation of more than $-40.5 \mathrm{~mm}$ and finished after only 30 trials of random length line bisection rightward deviation of only $+1.5 \mathrm{~mm}$. This positive regression of the deficit was clearly confirmed comparing the first three and the last three trials (the three first trials were outside the confidence interval computed for control subjects, whereas the thre trials lasts of this session fall inside). This recovery was observed without verbal feedback.
Figures 2 and 3 report results for Patient 1 and Patient 2, respectively.

\section{DISCUSSION}

Simple clinical observations following awake surgery to remove slow-growing tumors revealed that substantial recovery can occur within a few days (Bonnetblanc et al., 2006; Desmurget et al., 2007; Duffau, 2001; Duffau \& Capelle 2001a, 2001b; Duffau, Sichez, et al., 2000; Krainik et al., 2003). To document these recovery processes better and to determine their kinetics, we used a classical line bisection task to test two patients after a resection of a LGG invading the left parietal area.

The patients rapidly and substantially recovered from major right hemi-neglect in only 4 hours, 48 hours after their surgery. For both patients performance in line bisection tests were significantly better during the second session than during the previous session 4 hours earlier. The performance of Patient 1 tended to deteriorate during each session. This may have been explained by the patient becoming tired, as she explicitly stated during the 
first two sessions. The performance of the patients was not stabilized at the end of the second day after surgery and was different when tested several days later; however, the variability was smaller. This suggests that the network which compensated for the initial deficit was not itself stabilized, and that its capacity to recover function was not fully attained at the end of the second day. Interestingly, between each session and as a global tendency, Patient 2 seemed to deviate alternatively from the left to the right and then to the left again but the size of this oscillation seemed to be absorbed progressively. It may be that reciprocal inhibition became unbalanced between both hemispheres due to the removal of the left parietal area and may induce an alternation of attention between the right and left hemi-fields. This may be normalized progressively from the first to the last session.

Strikingly, we observed an ultra fast recovery: the performance of Patient 2 improved substantially within 7 minutes during the first testing session. Initially, Patient 2 deviated strongly leftward from the middle of the $25-\mathrm{cm}$ line but unconsciously compensated for this leftward deviation within 7 minutes, despite the variation of line lengths during the trials. This partial but substantial recovery occurred while the patient was simply performing the line bisection task without verbal feedback. The recovery was mostly retained and improved during the following testing sessions, although the patient continued to display consistent deviation 96 hours after surgery. Given such large fluctuations in performances of Patient 2, small sample size, and very short time interval, it is questionable whether true recovery has occurred. However, the fact that both patients show persistent leftward deviations after the first testing session does not play against the hypothesis of a true recovery since passive mechanisms would rather induce a constant and linear improvement correlated with time. This was clearly not the case between each session for both patients. In addition, the rapidity of the recovery itself and the fact that the deviations were smaller for the shorter lines precluded any potential effects of anesthesia, a local effect resembling diaschisis especially in the peri-tumorous tissue or pure motor impairments. Interestingly, the regression of symptoms was observed at two different temporal scales: a 'slow' rate post surgically and a 'fast' rate associated with practice (without verbal feedback) during individual testing sessions. This adaptation resembles that observed in prismatic adaptation (and motor adaptation in general) which similarly involves two different temporal scales: one slow between testing sessions and one fast during testing sessions (see Figure 4 in Gauthier, Blouin, Bourdin, \& Vercher, 2007). Interestingly, Luauté et al. (2009) using functional magnetic resonance imaging (fMRI) demonstrated that dynamic changes in brain activity following prismatic exposure occurred in the intraparietal sulcus, the parieto-occipital sulcus and the cerebellum. In our case, as for classical motor adaptation processes patients may not fully retain their performances from one session to the next. As observed on the recovery curves, they may need some recall in order to improve their performance at the level they had reached in the previous session. This important recall may explain why they are so fatigable but this need to be more clearly demonstrated.

These findings are consistent with processes unmasking redundant networks being responsible for the rapid plastic phenomenon. Unmasking processes have also been suggested by observations made using intra-cortical mapping. For instance, it was shown that during the surgery itself, a rapid functional reorganization in the sensory-motor system of the brain could occur within 15-60 minutes (Duffau, 2001, Duffau \& Capelle 2001a; Duffau, Sichez, et al. 2000, for a review see Desmurget et al., 2007; Duffau \& Capelle 2001b). In this case, the resection may induce a local increase in cortical excitability allowing latent intracortical connections to be unmasked (Duffau, 2001; Duffau, Sichez, et al., 2000). Interestingly, our observations were made after the resection and the recovery was associated with practice without verbal feedback. Possibly, latent redundant networks revealed by the resection processes participate in the functional recovery (Nii, Uematsu, Lesser, \& Gordon, 1996).

For healthy individuals, motor practice leads to rapid reorganizations in the motor area in the intact brain. Indeed, simple repetitions of thumb flexions can induce rapid cortical representational changes and the motor cortex can retain specific kinematic aspects of the practiced movement within as little as 15 to 30 minutes (Classen, Liepert, Wise, Hallett, $\&$ Cohen, 1998). It has been suggested that a rapid reorganization of motor maps could be regarded as a short-term memory for movements and be the first step of skill acquisition. Using fMRI, O'Shea, Johansen-Berg, Trief, Göbel, and Rushworth (2007) observed that transcranial magnetic stimulation $(1 \mathrm{~Hz})$ of the left dorsal premotor cortex $(\mathrm{PMd})$ was followed by a rapid compensatory increase in activity in the right PMd and connected medial 
premotor areas. Subsequent transcranial magnetic stimulation (TMS) of the reorganized right PMd disrupted performance suggesting that this pattern of functional reorganization had a causal role in preserving behavior after a neuronal challenge. Rapid plastic phenomena, within a few hours, were thus observed in the primary motor or the premotor cortex. In our experiment, the subjects rapidly recovered with practice independently of pure motor impairments and for a much more complex task with less movement repetitions than those employed in the experiment reported by Classen et al. Moreover, in their study, the representational changes occurring in the motor cortex were transient and disappeared after a few minutes.

One explanation for the rapid compensations we observed is that with the slow growth of the lesion, the contralesional hemisphere may have been progressively prepared, by the uncovering of contralesional redundant networks that have been slowly developing due to the gradually invading tumor, such that when the tumor was removed homologue networks could be rapidly unmasked. The following arguments are consistent with this hypothesis.

First, it could be suggested that the rapid recovery was due to some functions in the peri-tumorous tissue remaining in place and not being recruited because of a local effect resembling diaschisis. However, this interpretation does not seem plausible because of the rapidity of the recovery.

Second, DES performed just before and during the resection indicated the possible persistence of functional responses within the LGG (Danks, Aglio, Gugino, \& Black, 2000; Ojemann, Miller, \& Silbergeld, 1996; Skirboll et al., 1996), in the somatosensory motor cortex (Duffau et al., 2003b), in the left insular cortex (Duffau et al., 2000a) or in the left paralimbic cortex (Duffau, Taillandier, Gatignol, \& Capelle, 2006). As some functions which were not tested during the surgery can persist within the tumor, its removal could suddenly modify the transcallosal inhibition and modify the interhemispheric balance.

Third, this could have a more massive effect if the lesion was acute as in stroke, but the slow growth of the tumor may allow redundant networks to be better prepared for changes in the functional connectivity resulting from the surgery. It is possible that the compensations could occur anywhere in the brain and induce a progressive migration of the function ipsi or contralesionally. Indeed, various patterns of reorganizations involving perilesional, ipsilesional and contralesional areas have been observed before and after resections of LGG, some even in atypical areas (see Bonnetblanc et al., 2006, Desmurget et al., 2007; Duffau, 2005, 2008, for reviews). For resections of this size, with substantial evolution of the lesion, we speculate that the contralesional homologue is the best candidate to allow recovery of function because it probably contains the networks most similar to those removed. Krainik et al. (2004), using fMRI, observed the involvement of the contralesional homologue after the resection of the supplementary motor area. However, questions have been raised about the significance of these functional migrations: the recruitment of the intact contralesional homologues may reflect a decrease in transcallosal inhibition (Heiss, Thiel Kessler, \& Herholz, 2003; Netz, Ziemann, \& Homberg, 1995). A recent study rebuts these concerns by showing that TMS induces speech disruption when applied to the contralesional activation sites (Thiel et al., 2005).

It would be interesting to determine the neural correlations of this fast recovery using fMRI and TMS; such investigations would better elucidate the unmasking phenomenon. Indeed, it may be possible to demonstrate the role of the contralesional hemisphere using TMS.

Finally, our results have significant clinical implications. It is generally believed that visuoattentional deficits illustrated by hemineglect are widely mediated by lesions of the right parietal cortex (Kandel et al., 2000; Purves et al., 2008; Robertson \& Marshall 1993). We show that for patients with a left invading tumor, line bisection tests are required before, during and after any awake surgery to help plan the surgery, control the quality of the resection, and also plan patient rehabilitation.

\footnotetext{
Original manuscript received 14 July 2010 Revised manuscript accepted 14 October 2010 First published online day/month/year
}

\section{REFERENCES}

Berger, M. S., \& Rostomily, R. C. (1997). Low grade gliomas: Functional mapping resection strategies, extent of resection, and outcome. Journal of NeuroOncology, 34, 85-101.

Bonnetblanc, F., Desmurget, M., \& Duffau, H. (2006). Low grade gliomas and cerebral plasticity: Fundamental and clinical implications. Medecine Science (Paris), 22, 389-394.

Bowen, A., McKenna, K., \& Tallis, R. C. (1999). Reasons for variability in the reported rate of occurrence of unilateral spatial neglect after stroke. Stroke, 30, 1196-1202. 
Chain, F., Leblanc, M., Chedru, F., \& L'Hermitte, F. (1979). Negligence visuelle dans les lesions posterieures de l'hemisphere gauche. Rev Neurol., 135, $105-126$

Chedru, F., Leblanc, M., \& L'Hermitte, F. (1973). Visual searching in normal and brain-damaged subjects (a contribution to the study of uni-lateral attention). Cortex, 9, 94-111.

Classen, J., Liepert, J., Wise, S. P., Hallett, M., \& Cohen, L. G. (1998). Rapid plasticity of human cortical movement representation induced by practice. Journal of Neurophysiology, 79, 1117-1123.

Danks, R. A., Aglio, L. S., Gugino, L. D., \& Black, P. M. (2000). Craniotomy under local anesthesia and monitored conscious sedation for the resection of tumors involving eloquent cortex. Journal of Neuro-Oncology, 49, 131-139.

Denes, G., Semenza, C., Stoppa, E., \& Lis, A. (1982). Unilateral spatial neglect and recovery from hemiplegia: A follow-up study. Brain, 105, 543-552.

Desmurget, M., Bonnetblanc, F., \& Duffau, H. (2007). Contrasting acute and slow-growing lesions: A new door to brain plasticity. Brain, 130, 898-914.

Duffau, H. (2001). Acute functional reorganisation of the human motor cortex during resection of central lesions: A study using intraoperative brain mapping. Journal of Neurology, Neurosurgery and Psychiatry, 70, 506-513.

Duffau, H. (2004). Peroperative functional mapping using direct electrical stimulations. Methodological considerations. Neurochirurgie, 50, 474-483.

Duffau, H. (2005). Lessons from brain mapping in surgery for low-grade glioma: Insights into associations between tumour and brain plasticity. The Lancet Neurology, 4, 476-486.

Duffau, H. (2008). Brain plasticity and tumors. Advanced Technological Standards in Neurosurgery, 33, 3-33.

Duffau, H., \& Capelle, L. (2001a). Functional recuperation following lesions of the primary somatosensory fields. Study of compensatory mechanisms. Neurochirurgie, 47, 557-563.

Duffau, H, \& Capelle, L. (2001b). Functional recuperation after resection of gliomas infiltrating primary somatosensory fields. Study of perioperative electric stimulation. Neurochirurgie, 47, 534-541.

Duffau, H., Capelle, L., Lopes, M., Faillot, T., Sichez, J. P., \& Fohanno, D. (2000). The insular lobe: Physiopathological and surgical considerations. Neurosurgery, 47, 801-810.

Duffau, H., Sichez, J. P., \& Lehericy, S. (2000). Intraoperative unmasking of brain redundant motor sites during resection of a precentral angioma: Evidence using direct 55 cortical stimulation. Annals of Neurology, 47, 132-135.

Duffau, H., Bauchet, L., Lehericy, S., \& Capelle, L. (2001). Functional compensation of the left dominant insula for language. Neuroreport, 12, 2159-2163.

Duffau, H., Capelle, L., Denvil, D., Sichez, N., Gatignol, P., Taillandier, L., et al. (2003a). Usefulness of intraoperative electrical subcortical mapping during surgery for low-grade gliomas located within eloquent brain regions: Functional results in a consecutive series of 103 patients. Journal of Neurosurgery, 98, 764-778.
Duffau, H., Capelle, L., Denvil, D., Sichez, N., Gatignol, P., Lopes, M., et al. (2003b). Functional recovery after surgical resection of low grade gliomas in eloquent brain: Hypothesis of brain compensation. Journal of Neurology, Neurosurgery and Psychiatry, 74, 901-907.

Duffau, H., Taillandier, L., Gatignol, P., \& Capelle, L. (2006). The insular lobe and brain plasticity: Lessons from tumor surgery. Clinical Neurology and Neurosurgery, 108, 543-548.

Duffau, H., Gatignol, P., Mandonnet, E., Capelle, L., \& Taillandier, L. (2008). Contribution of intraoperative subcortical stimulation mapping of language pathways: A consecutive series of 115 patients operated on for a WHO grade II glioma in the left dominant hemisphere. Journal of Neurosurgery, 109, 461-471.

Finger, S. (1978). Lesion momentum and behavior. In S. Finger (Ed.), Recovery from brain damage: Research and theory (pp. 135-164). New York, NY: Plenum Press.

Finger, S., \& Stein, D. (1982). Brain damage and recovery: Research and clinical perspectives. Orlando, FL: Academic Press.

Gauthier, G. M., Blouin, J., Bourdin, C., \& Vercher, J. L. (2007). Adaptive control: A review of the ability to acquire and maintain high sensorimotor performance. Computers in Biology and Medicine, 37, 989-1000.

Heiss, W. D., Thiel, A., Kessler, J., \& Herholz, K. (2003). Disturbance and recovery of language function: Correlates in PET activation studies. Neuroimage, 20 (Suppl. 1), S42-49.

Kandel, E. R., Schwartz, J. H., \& Jessell, T. M. (Eds.) (2000). Principles of neural science (4th ed.). New York, NY: McGraw-Hill.

Keles, G. E., \& Berger, M. S. (2004). Advances in neurosurgical technique in the current management of brain tumors. Seminars in Oncology, 31, 659-665.

Keles, G. E., Lundin, D. A., Lamborn, K. R., Chang, E. F., Ojemann, G., \& Berger, M. S. (2004). Intraoperative subcortical stimulation mapping for hemispherical perirolandic gliomas located within or adjacent to the descending motor pathways: Evaluation of morbidity and assessment of functional outcome in 294 patients. Journal of Neurosurgery, 100, 369-375.

Krainik, A., Lehericy, S., Duffau, H., Capelle, L., Chainay, H., Cornu, P., et al. (2003). Postoperative speech disorder after medial frontal surgery: Role of the supplementary motor area. Neurology, 60, 587594.

Krainik, A., Duffau, H., Capelle, L., Cornu, P., Boch, A. L., Mangin, J. F., et al. (2004). Role of the healthy hemisphere in recovery after resection of the supplementary motor area. Neurology, 62, 1323-1332.

Liepmann, H. (1920). Apraxie. Ergebnisse der gesamten Medizin, 1, 516-543.

Luauté, J., Schwartz, S., Rossetti, Y., Spiridon, M., Rode, G., Boisson, D., \& Vuilleumier, P. (2009). Dynamic changes in brain activity during prism adaptation. Journal of Neuroscience, 29, 169-178.

Mandonnet, E., Delattre, J. Y., Tanguy, M. L., Swanson, K. R., Carpentier, A. F., Duffau, H., et al. (2003). 
Continuous growth of mean tumor diameter in a subset of grade II gliomas. Annals of Neurology, 53, 524-528.

Netz, J., Ziemann, U., \& Homberg, V. (1995). Hemispheric asymmetry of transcallosal inhibition in man. Experimental Brain Research, 104, $527-533$

Nii, Y., Uematsu, S., Lesser, R. P., \& Gordon, B. (1996). Does the central sulcus divide motor and sensory functions? Cortical mapping of human hand areas as revealed by electrical stimulation through subdural grid electrodes. Neurology, 46, 360-367.

Ogden, J. A. (1985). Anterior-posterior interhemispheric differences in the loci of lesions producing visual hemineglect. Brain Cognition, 4, 59-75.

Ojemann, G., Ojemann, J., Lettich, E., \& Berger, M. (1989). Cortical language localization in left, dominant hemisphere. An electrical stimulation mapping investigation in 117 patients. Journal of Neurosurgery, 71, 316-326.

Ojemann, J. G., Miller, J. W., \& Silbergeld, D. L. (1996). Preserved function in brain invaded by tumor. Neurosurgery, 39, 253-258.

O'Shea, J., Johansen-Berg, H., Trief, D., Göbel, S., \& Rushworth, F. S. (2007). Functionnally specific reorganization in human premotor cortex. Neuron, 54, 479-490.

Purves, D., Brannon, E. M., Cabeza, R., Huettel, S. A., LaBar, K. S., Platt, M. L., \& Woldorff, M. G. (Eds.) (2008). Principles of cognitive neuroscience. Sunderland, MA: Sinauer Associates.
Robertson, I., Marshall, J. (Eds.), Unilateral neglect clinical and experimental studies (pp. 3-25). Hove, England: Lawrence Erlbaum Associates.

Sanai, N., Mirzadeh, Z., \& Berger, M. S. (2008). Functional outcome after language mapping for glioma resection. New England Journal of Medicine, 358, 18-27.

Stone, S. P., Wilson, B., Wroot, A., Halligan, P. W., Lange, L. S., Marshall, J. C., \& Greenwood, R. J. (1991). The assessment of visuo-spatial neglect after acute stroke. Journal of Neurology, Neurosurgery and Psychiatry, 54, 345-350.

Sunderland, A., Wade, D. T., \& Langton-Hewer, R. (1987). The natural history of visual neglect after stroke: Indications from two methods of assessment. Journal of International Disability Studies, 9, 55-59.

Taphoorn, M. J., \& Klein, M. (2004). Cognitive deficits in adult patients with brain tumours. The Lancet Neurology, 3, 159-168.

Thiebaut de Schotten, M., Urbanski, M., Duffau, H., Volle, E., Levy, R., Dubois, B., et al. (2005). Direct evidence for a parietal-frontal pathway subserving spatial awareness in humans. Science, 309, 2226-2228.

Thiel, A., Habedank, B., Winhuisen, L., Herholz, K., Kessler, J., Haupt, W. F., et al. (2005). Essential language function of the right hemisphere in brain tumor patients. Annals of Neurology, 57, 128-131.

Walker, D. G., \& Kaye, A. H. (2003). Low grade glial neoplasms. Journal of Clinical Neuroscience, 10, 1-13. 